\title{
Identification of proteins with different abundance associated with cell migration and proliferation in leiomyoma interstitial fluid by proteomics
}

\author{
BLENDI URA $^{1}$, FEDERICA SCRIMIN ${ }^{1}$, CINZIA FRANCHIN ${ }^{2,3}$, GIORGIO ARRIGONI ${ }^{2,3}$, \\ DANILO LICASTRO $^{4}$, LORENZO MONASTA ${ }^{1}$ and GIUSEPPE RICCI ${ }^{1,5}$
}

${ }^{1}$ Institute for Maternal and Child Health, IRCCS 'Burlo Garofolo', I-34137 Trieste; ${ }^{2}$ Department of Biomedical Sciences,
University of Padova, I-35122 Padova; ${ }^{3}$ Proteomics Center, University of Padua and Padua Hospital, I-35129 Padova;
${ }^{4}$ Consortium for Molecular Biomedicine Genomics, Area Science Park, Basovizza, I-34149 Trieste;
${ }^{5}$ Department of Medical, Surgery and Health Sciences, University of Trieste, I-34128 Trieste, Italy

Received November 4, 2016; Accepted January 26, 2017

DOI: $10.3892 / \mathrm{ol} .2017 .5943$

\begin{abstract}
Uterine leiomyoma is the most common female reproductive tract benign tumor. Little is known about protein composition and changes in the leiomyoma interstitial fluid (IF). The present study focused on changes in protein abundance in the IF of leiomyoma. Leiomyoma IFs and adjacent myometrial IFs were obtained and analyzed by two-dimensional electrophoresis (2-DE) coupled with mass spectrometry and western blotting for 2-DE data validation. A total of 25 unique proteins were observed to change significantly $(\mathrm{P}<0.05)$. Of these proteins with different abundance, 22 had not been previously identified in leiomyoma IF. In silico analysis predicted that three of these proteins were secreted via classical mechanisms, while 22 were secreted via non-classical mechanisms. Ingenuity Pathway Analysis identified 17 proteins associated with cellular migration and proliferation. Among these, phosphoglycerate mutase 1 had not been previously associated with leiomyoma. The abundance of seven proteins was further validated by western blotting. A comparative proteomic approach identified a number of proteins associated with cellular migration and proliferation, with changes in abundance in IF likely to be involved in tumor development. Further studies will be required to investigate the role of these proteins in leiomyoma IF and their possible association with tumor development and growth.
\end{abstract}

Correspondence to: Dr Lorenzo Monasta, Institute for Maternal and Child Health, IRCCS 'Burlo Garofolo', Via del'Istria 65/a, I-34137 Trieste, Italy

E-mail: lorenzo.monasta@burlo.trieste.it

Key words: leiomyoma, myometrium, 2-D electrophoresis, proteomics, interstitial fluid, mass spectrometry, secreted protein

\section{Introduction}

Uterine leiomyoma is the most common female reproductive tract benign tumor (1). This tumor is responsible for severe health problems, including pain, infertility, repetitive pregnancy loss and, most importantly, heavy uterine bleeding (2). No effective medical treatment is currently available, due to the poor understanding of the exact molecular pathways involved in its physiopathology (3). Ovarian hormones, growth factors, retinoic acid, vitamin D, transforming growth factor- $\beta /$ Smad and genetic factors are associated with tumor development (4,5). Leiomyomas are characterized by a hyperproduction of extracellular matrix (ECM), which is constituted predominantly by collagens and other proteoglycans (6). The ECM components serve a key role in the regulation of migration, proliferation and differentiation of leiomyomas (6).

The interstitial fluid (IF) is an extracellular fluid bathing and surrounding the tissue cells, and is closely associated with pathological changes in the tissues. In addition to nutrients and oxygen, IF contains proteins secreted by tumors via classical and non-classical secretory pathways, as well as proteins released by extracellular vesicles (7). Several studies have shown that tumor growth is not only regulated by the malignancy of the tumor cells, but is also associated with several factors that are present in the tumor microenvironment, including cytokines, electrolytes and other secreted proteins (8). The IF flow movement may influence the degree of viscosity and elasticity of the ECM, thus modifying the intercellular communication and determining an increased mitotic activity (9). Collagen and other proteoglycans such as lumican (LUM) induce the activation of the integrin signaling pathway, which serves a key role in cytoskeleton reorganization, cell motility and proliferation (6). Early cytogenetic alterations are allegedly insufficient for tumor development; thus, additional alterations of the MAPK/ERK signaling pathway appear to be crucial (3).

For the reasons described above, the identification of changes in protein abundance in leiomyoma IF may be useful for the understanding of the tumor physiopathology. Proteomic studies on leiomyomas, carried out to identify dysregulated 
proteins, have been conducted in two-dimensional electrophoresis (2-DE) coupled with mass spectrometry (MS) (10) and gel electrophoresis liquid chromatography-MS (11). Our previous proteomic studies on leiomyoma tissue identified several dysregulated proteins involved in metabolic processes, oxidative stress and cell migration $(12,13)$. In a previous study, seven proteins with changes in abundance were identified in the IF of leiomyomas (14). To date, no other proteomic study has been conducted on the IF of leiomyomas.

The aim of the present study was to analyze the proteome of leiomyoma and myometrium IF in order to identify changes in the abundance of proteins potentially associated with tumor growth. A comparative proteomic approach identified 17 proteins associated with cellular migration and proliferation with different abundance in leiomyoma IF.

\section{Materials and methods}

Samples. Tissue samples were obtained from eight premenopausal female patients who underwent hysterectomy for symptomatic uterine leiomyoma. Samples were collected between February 2015 and June 2015 at the Institute for Maternal and Child Health, IRCCS 'Burlo Garofolo' (Trieste, Italy). The procedures complied with the Declaration of Helsinki and were approved by the Technical and Scientific Committee of the Institute for Maternal and Child Health, IRCCS 'Burlo Garofolo' of Trieste, Italy (approval no. RC 19/08). All subjects signed a written informed consent. The patients did not receive any medication (including hormonal therapy) during the three months prior to surgery. All samples were obtained during the proliferative phase. Two samples were collected from each patient: One from the central area of the leiomyoma and one from the unaffected myometrium. The median age of the patients was 46 years (range, 42-52 years).

Preparation of IF. The IF was obtained as previously described $(14,15)$. Two samples $(300 \mathrm{mg}$ each) were collected from each patient: One from the unaffected myometrium and one from the central area of the leiomyoma. All leiomyomas were confirmed histologically as benign ordinary leiomyomas. Leiomyoma and myometrium tissues were dissected into $0.1-0.3-\mathrm{cm}^{3}$ pieces and rinsed three times with sterile PBS containing a protease inhibitor mixture $(2 \mathrm{mM}$ phenylmethylsulfonyl fluoride, $1 \mathrm{mM}$ benzamidine, $1 \mathrm{mM}$ EDTA and $10 \mathrm{mM} \mathrm{NaF}$ ). The samples were incubated with PBS in a humidified $\mathrm{CO}_{2}$ incubator for $1 \mathrm{~h}$ at $37^{\circ} \mathrm{C}$. The supernatant was centrifuged at $5,000 \times \mathrm{g}$ for $20 \mathrm{~min}$ at $4^{\circ} \mathrm{C}$, followed by centrifugation at $15,000 \times \mathrm{g}$ for $30 \mathrm{~min}$ at $4^{\circ} \mathrm{C}$ to remove cell debris. Approximately $5 \mathrm{ml}$ of supernatant was desalted and concentrated using an Ultrafree-4 Centrifugal Filter Unit (EMD Millipore, Billerica, MA, USA) with a molecular mass cut-off of $10 \mathrm{kDa}$ at $4,000 \mathrm{x} g$ at $25^{\circ} \mathrm{C}$ until the remaining volume reached $200 \mu \mathrm{l}$. The obtained supernatant was designated as the IF, and the protein content of the supernatant was determined by Bradford assay.

2-DE and image analysis. For 2-DE, $700 \mu \mathrm{g}$ of proteins from each IF sample were denatured in $350 \mu \mathrm{l}$ of dissolution buffer [7 $\mathrm{M}$ urea, $2 \mathrm{M}$ thiourea, 4\% 3-[(3-cholamidopropyl) dimethylammonio]-1-propanesulfonate, $40 \mathrm{mM}$ Tris, $65 \mathrm{mM}$ dithiothreitol (DTT) and 0.24\% Bio-Lyte 3/10 (Bio-Rad Laboratories, Inc., Hercules, CA, USA)] and $7 \mu \mathrm{g}$ bromophenol blue. ReadyStrip ${ }^{\mathrm{TM}} \mathrm{pH}$ 3-10 non-linear (NL) 18-cm immobilized $\mathrm{pH}$ gradient (IPG) strips (Bio-Rad Laboratories, Inc.) were rehydrated in a dissolution buffer at $50 \mathrm{~V}$ for $12 \mathrm{~h}$ at $20^{\circ} \mathrm{C}$, and isoelectric focusing (IEF) was performed in a PROTEAN IEF Cell (Bio-Rad Laboratories, Inc.) using the following protocol: $150 \mathrm{~V}$ for $1 \mathrm{~h}, 250 \mathrm{~V}$ for $2 \mathrm{~h}$, increase from $250 \mathrm{~V}$ to $10,000 \mathrm{~V}$ in $2 \mathrm{~h}, 10,000 \mathrm{~V}$ for $10 \mathrm{~h}$ and $10,000 \mathrm{~V}$ for $6 \mathrm{~h}$, for a total of $170,000 \mathrm{~V} / \mathrm{h}$. Serial incubations were performed following IEF. First, the IPG strips were equilibrated for $20 \mathrm{~min}$ in an equilibration buffer [6 $\mathrm{M}$ urea, 2\% SDS, $50 \mathrm{mM}$ Tris- $\mathrm{HCl}$ (pH 8.8), 30\% glycerol and 1\% DTT] and then in another equilibration buffer containing $4 \%$ iodoacetamide instead of DTT. For the second dimension, the equilibrated IPG strips were transferred to a $12 \%$ polyacrylamide gel $(19 \times 20 \mathrm{~cm})$. The SDS gels were subjected to electrophoresis on a Protean II XL Cell (Bio-Rad Laboratories, Inc.) at $200 \mathrm{~V}$ constant voltage, until the bromophenol blue band reached the bottom of the gel. Upon electrophoresis, the gels were fixed in $40 \%$ methanol and $10 \%$ acetic acid, and then stained for $48 \mathrm{~h}$ with colloidal Coomassie Brilliant Blue. Triple experimental replicates were performed per sample. Molecular masses were determined by comparison with Precision Plus Protein ${ }^{\mathrm{TM}}$ Prestained Standards (Bio-Rad Laboratories, Inc.), covering a range between 10 and $250 \mathrm{kDa}$. 2-DE gels were scanned with a Molecular Imager PharosFX system and analyzed using the Proteomweaver software version 4.0 (both from Bio-Rad Laboratories, Inc.).

Quantification of spot levels. Spot quantification was performed as previously described (12). 2-DE image analysis was performed using the Proteomweaver 4 software. The analysis was performed by matching all gels from eight myometrial IFs and eight leiomyoma IFs. Differences were considered to be significant when the ratio of the mean percentage of relative volume $(\% \mathrm{~V})$, determined as $\% \mathrm{~V}=\mathrm{V}_{\text {single spot }} / \mathrm{V}_{\text {total spot }}$, was 1.5 -fold for upregulated and 0.6 -fold for downregulated proteins, and satisfied the non-parametric Wilcoxon signed-rank test for matched samples $(\mathrm{P}<0.05)$. Fold change was calculated as the ratio between the mean $\% \mathrm{~V}$ of the uterine leiomyoma and that of the normal myometrium.

Trypsin digestion and matrix-assisted laser desorption/ionization (MALDI)-time-of-flight (TOF)/TOF analysis. Spots from 2-DE gels were thoroughly washed with alternating changes of $50 \mathrm{mM} \mathrm{NH}_{4} \mathrm{HCO}_{3}$ and acetonitrile (ACN), and then dried under vacuum. A total of $5 \mu \mathrm{l}$ of sequencing-grade modified trypsin $\left(12.5 \mathrm{ng} / \mathrm{ml}\right.$ in $50 \mathrm{mM} \mathrm{NH}_{4} \mathrm{HCO}_{3}$; Promega Corporation, Madison, WI, USA) were added to each gel spot, and samples were kept in ice for $30 \mathrm{~min}$ to allow the gel to completely rehydrate. A total of $50 \mathrm{mM} \mathrm{NH}_{4} \mathrm{HCO}_{3}$ was added to each sample until gel pieces were completely covered, and digestion was carried out overnight at $37^{\circ} \mathrm{C}$. Peptides were extracted with three changes of $50 \%$ ACN $/ 0.1 \%$ trifluoroacetic acid (TFA; Honeywell Riedel-de Haën, Seelze, Germany), dried under vacuum and then dissolved in $10 \mu \mathrm{l}$ of $0.1 \%$ TFA. One $\mu \mathrm{l}$ of each sample was mixed with an equal volume of matrix $[\alpha$-cyano-4-hydroxycinnamic acid (Sigma-Aldrich; 


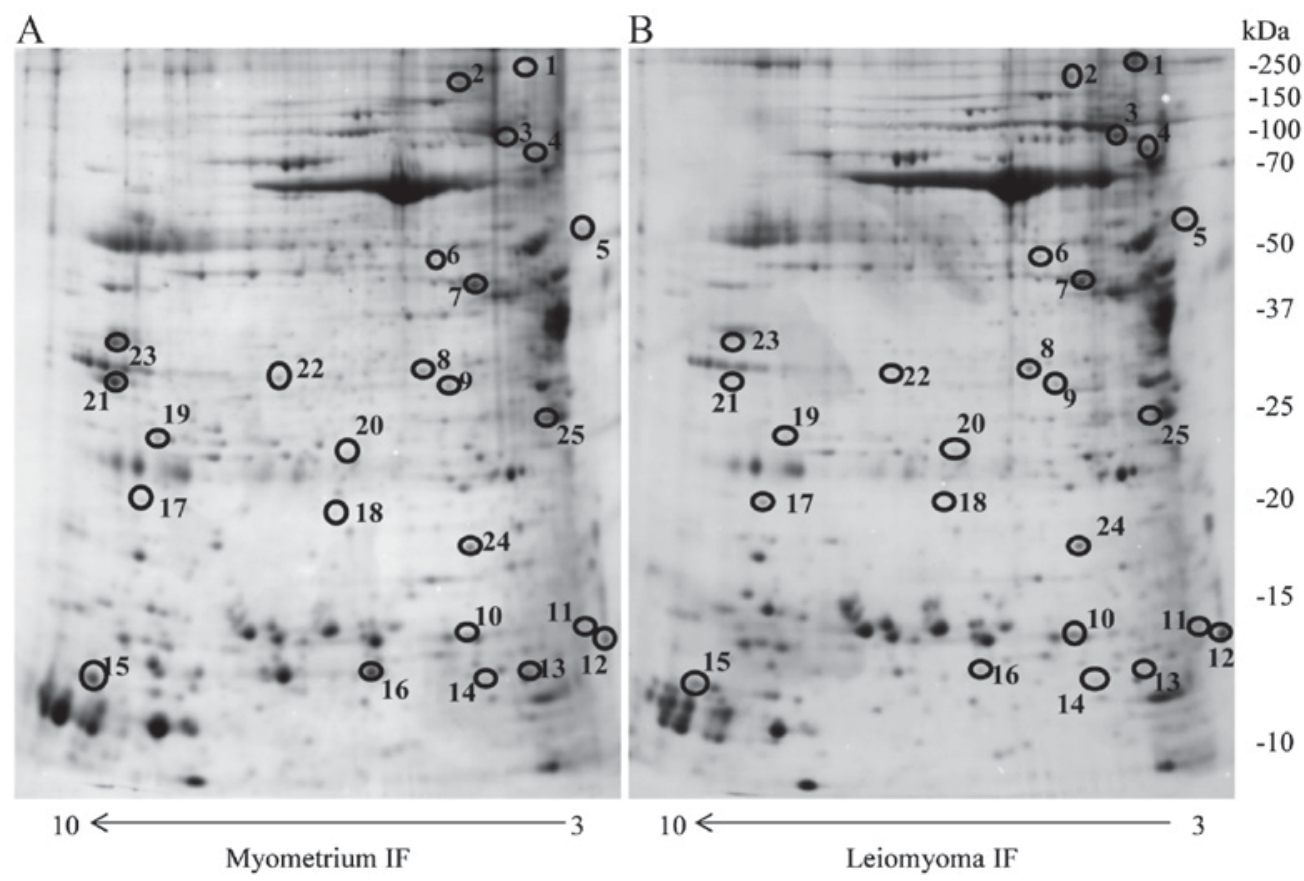

Figure 1. Two-dimensional electrophoresis map of the (A) normal myometrium IF and (B) leiomyoma IF proteome. Immobilized pH gradient pH 3-10 non-linear strips were used for the first dimension and $12 \%$ polyacrylamide gels were used for the second dimension. IF, interstitial fluid. Circles indicate different proteins identified.

Merck KGaA, Darmstadt, Germany), $5 \mathrm{mg} / \mathrm{ml}$ in $70 \%$ $\mathrm{ACN} / 0.1 \% \mathrm{TFA}]$, and $0.8 \mu \mathrm{l}$ of the resulting solution was spotted onto a stainless steel MALDI target plate (AB SCIEX, Framingham, MA, USA). Mass spectrometry (MS)/MS analysis was performed on a 4800 MALDI-TOF/TOF Analyzer (AB SCIEX) in a data-dependent mode: For each sample, a full MS scan was acquired, followed by MS/MS spectra of the 10 most intense signals.

Data were converted into Mascot generic format files and analyzed with Proteome Discoverer software (version 1.4; Thermo Fisher Scientific, Inc., Waltham, MA, USA) interfaced to a Mascot search engine (version 2.2.4; Matrix Science Ltd., London, UK). Data were searched against the human section of the UniProt database (www.uniprot.org; version 20,150,401; 90,411 sequences). Enzyme specificity was set to trypsin with $\leq 1$ missed cleavage. Tolerance was set to $50 \mathrm{ppm}$ and $0.3 \mathrm{Da}$ for parent and fragment ions, respectively. Carbamidomethylation of cysteines was set as a fixed modification, while methionine oxidation was set as a variable modification. The software calculates the false discovery rate based on the search against a randomized database. Proteins were considered as positively identified if $\geq 2$ unique peptides were identified for each protein with high confidence $(\mathrm{q}<0.01)$.

Prediction of secreted protein candidates. SecretomeP 2.0 (Center for Biological Sequence Analysis, Technical University of Denmark, Lyngby, Denmark) was used to classify proteins as following a classical (proteins with signal peptide) or non-classical (proteins without signal peptide but whose neural network score exceeded the normal threshold of 0.5) secretory pathway. To verify the possible release of proteins by exosomes and microvesicles, manual annotation on the Exocarta (exocarta.org) and Vesiclepedia (microvesicles.org/ index.html) databases was used.
Western blotting. Western blot analysis was performed as previously described (16). An equal amount of protein to that used for 2-DE analysis $(30 \mu \mathrm{g})$ was separated by $12 \%$ SDS-PAGE and then transferred to a nitrocellulose membrane. The residual binding sites on the membrane were blocked by treatment with 5\% skim milk in TBS containing $0.05 \%$ Tween-20 (TBST) for $2 \mathrm{~h}$ at room temperature. Next, the membrane was incubated overnight at $4^{\circ} \mathrm{C}$ with 1:500-diluted primary rabbit polyclonal antibody against cellular retinoic acid binding protein 2 (CRABP2; cat. no. SAB2100477), 1:300-diluted primary rabbit polyclonal antibody against phosphoglycerate mutase 1 (PGAM1; cat. no. WH0005223M1), 1:800-diluted primary rabbit polyclonal antibody against aldolase A (ALDOA; cat. no. SAB1410385), 1:700-diluted primary rabbit polyclonal antibody against LUM (cat. no. SAB1401232), 1:500-diluted primary rabbit polyclonal antibody against annexin A2 (ANXA2; cat. no. SAB1410612), 1:1,000-diluted primary rabbit polyclonal antibody against calreticulin (CALR; cat. no. AV30114) and 1:200-diluted primary rabbit polyclonal antibody against peroxiredoxin (PRDX1; cat. no. HPA007730) (all antibodies were supplied by Sigma-Aldrich; Merck KGaA). The membrane was washed three times in TBST for $10 \mathrm{~min}$, and then incubated for $2 \mathrm{~h}$ at $4^{\circ} \mathrm{C}$ with a horseradish peroxidase-conjugated anti-rabbit immunoglobulin $\mathrm{G}$ antibody (cat. no. A8275) (Sigma-Aldrich; Merck KGaA) at 1:3,000 dilution. Protein expression was visualized by chemiluminescence (SuperSignal West Pico Chemiluminescent Substrate; Thermo Fisher Scientific, Inc.), and the intensity of the signals was quantified by VersaDoc Imaging System (Bio-Rad Laboratories, Inc.). The intensities of the immunostained bands were normalized with the protein intensities measured by Red Ponceau S reagent (Sigma-Aldrich; Merck KGaA) from the same blot. 


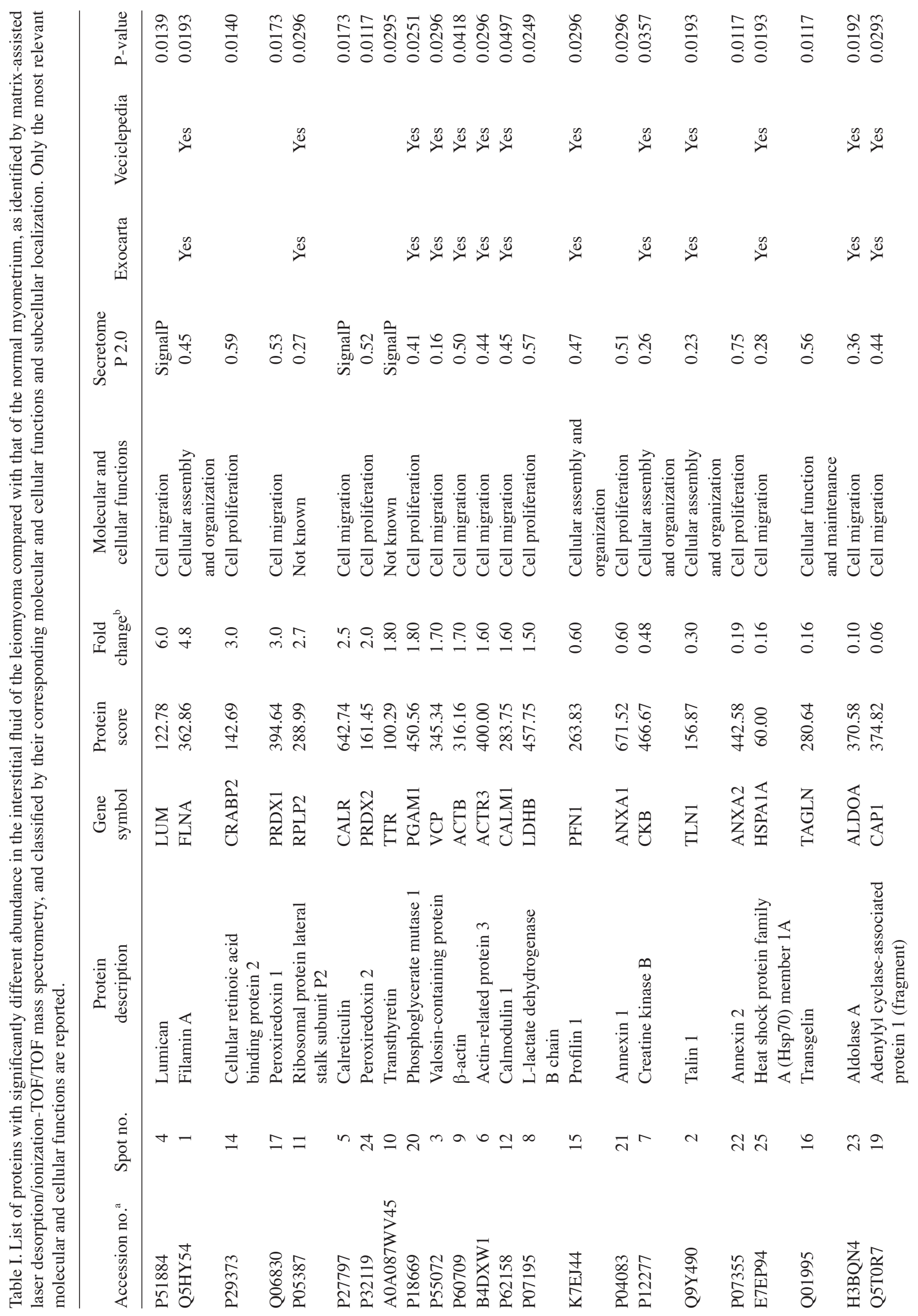


Ingenuity Pathway Analysis (IPA). Changes in protein abundance identified by MS in leiomyoma IF were analyzed by IPA (Qiagen GmbH, Hilden, Germany) as previously described (16). Selected genes were incorporated in canonical pathways and bio-functions, and were used to generate biological networks. For the filter summary, only associations where confidence was high (predicted) or that had been experimentally observed were considered. As cut-off, and adjusted $\mathrm{P}<0.05$ value was used. The network score was based on the hypergeometric distribution, and was calculated with a two-tailed Fisher's exact test. Since the majority of proteins identified participated in multiple processes, only the most relevant ones were reported.

Statistical analysis. Statistical analyses were carried out with the non-parametric Wilcoxon signed-rank test for matched samples for both $2-\mathrm{DE}$ and western blot data. $\mathrm{P}<0.05$ was considered to indicate a statistically significant difference. All analyses were conducted with Stata/IC 14.1 for Windows (StataCorp LP, College Station, TX, USA).

\section{Results}

2-DE and $M S$. In the present study, the IF of eight leiomyomas and myometria from eight premenopausal women were resolved using 2-DE. Approximately 2,000 protein spots were detected in the 2-DE gels for the two types of IFs. Correlation of gel-pairs performed with an average matching efficiency of $\sim 75 \%$. The analysis revealed 25 protein spots with different abundance in the IF of leiomyoma compared with that of the IF of the myometrium. Only protein spots with fold change in $\% \mathrm{~V} \geq 1.5$ or $\leq 0.6$ in intensity that were significant $(\mathrm{P}<0.05)$ were considered (Fig. 1). These proteins were identified by MALDI-TOF/TOF and database search against the human section of the UniProt database (version 20150401; 90,411 sequences) (Table I).

In addition, proteins reported in our previous study, including desmin (DES), transgelin (TAGLN), serpin family A member 1 (SERPINA1), lamin A/C (LMNA), actinin $\alpha 1$ (ACTN1), PRDX2 and heat shock protein family A (Hsp70) member 1A (HSPA1A), were also identified (14). Of these proteins, TAGLN, PRDX2 and HSPA1A were significant, while DES, SERPINA1 (more abundant in the leiomyoma compared with in the myometrium), LMNA and ACTN1 (less abundant in the leiomyoma compared with in the myometrium) were not significant, and thus were not investigated further.

In total, 22 of the 25 proteins with different abundance reported in the present study have not been previously identified in the leiomyoma IF. Furthermore, among the identified proteins, one (namely PGAM1) has never been previously associated with leiomyoma.

Prediction of protein secretion and functional analysis of the leiomyoma proteome. To define if the 25 identified proteins were secreted by the classical or non-classical secretory pathway, the SignalIP version 4.1 (www.cbs.dtu.dk/services/ SignalP) and SecretomeP 2.0 (www.cbs.dtu.dk/services/ SecretomeP) prediction servers were used. In addition, the Vesiclepedia and Exocarta databases, which contain proteins identified in extracellular vesicles and exosomes released by non-classical secretory pathways respectively, were used. Out 

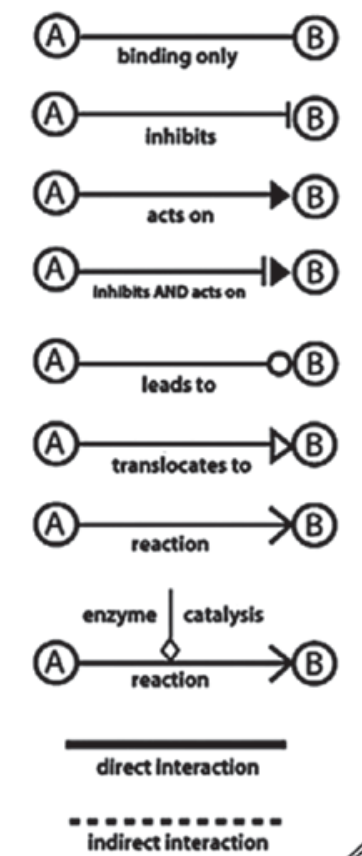

Figure 2. Top-scored molecular networks involving proteins with different abundance in the leiomyoma interstitial fluid, according to the results of Ingenuity Pathway Analysis software. Proteins in the network are represented by their gene symbols.

of the 25 proteins, three were assigned to the classical secretory pathway predicted by their signal peptide: LUM, CALR and transthyretin. The remaining proteins were predicted to be secreted by non-classical pathways to the extracellular space through various mechanisms (Table I).

$I P A$. Proteins with significantly different abundance in the IF of leiomyoma compared with that of the myometrium were used in the core analysis with IPA software. The analysis revealed that the identified proteins were associated with cellular migration, proliferation, assembly, organization, function and maintenance.

A total of 17 proteins were observed to be associated with cellular migration and proliferation according to the IPA prediction (Table I). Fig. 2 shows a graphical representation of the predicted interaction between the proteins with significantly different abundance identified in the present study. This network (score, 56) includes 25 identified proteins involved in neurological diseases, cellular migration, and skeletal and muscular disorders. The representative interaction network suggests that the proteins with changes in abundance identified in the present study may influence the activity of other proteins within the network.

Immunohistochemical study of protein expression. Proteins with different abundance in the leiomyoma IF (namely LUM, PRDX, CALR, CRABP2, ANXA2, ALDOA and PGAM1) were further validated by western blotting in five leiomyomas (IF) vs. normal myometrium (IF) (Fig. 3). The abundance of LUM, PRDX1, CALR, CRABP2 and PGAM1 was significantly 

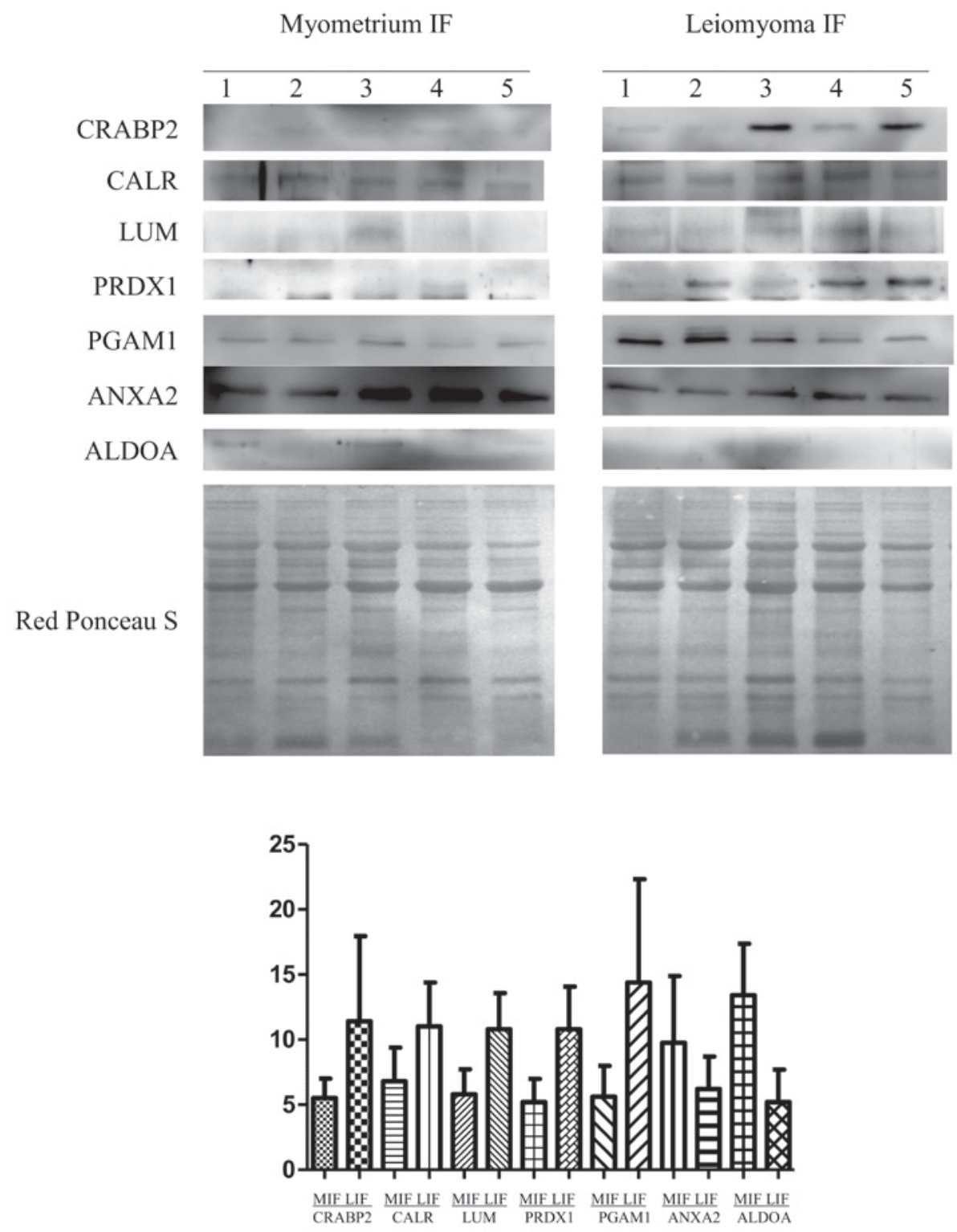

Figure 3. Western blot analysis of CRABP2, CALR, LUM, PRDX1, PGAM1, ANXA2 and ALDOA in paired MIF and LIF. The intensity of the immunostained bands was normalized against the total protein intensities measured from the same blot stained with Red Ponceau S. The bar graph shows the relative expression (band density) of proteins in the MIF and LIF. The results are shown as a histogram (mean) with error bars representing the standard deviation. All differences were observed to be significant (Wilcoxon signed-rank test for matched samples, P<0.05). MIF, myometrium IF; LIF, leiomyoma IF; IF, interstitial fluid; CRABP2, cellular retinoic acid binding protein 2; CALR, calreticulin; LUM, lumican; PRDX, peroxiredoxin; PGAM1, phosphoglycerate mutase 1; ANXA2; annexin A2; ALDOA, aldolase A.

higher in leiomyomas than in the myometrium, while for ANXA2 and ALDOA, their abundance was significantly lower in leiomyomas with respect to that in the myometrium, thus confirming the results obtained from the 2-DE analysis. To normalize the results of the western blot analysis, the total protein content of each sample, as determined by Red Ponceau S staining, was used. According to our previous results (12), proteins encoded by housekeeping genes (i.e., $\beta$-actin and tubulin) are usually upregulated in leiomyomas and, therefore, not adequate to be used as controls for normalization.

\section{Discussion}

In tumor diseases, secreted proteins create conditions favorable to the disorder, promoting tumor progression and growth (17).
Proteomic studies are essential for the acquisition of knowledge about the qualitative and quantitative composition of the IF of the cell, and may be crucial to understand the biology of cell interaction, proliferation, differentiation, communication and migration. In a previous study, seven proteins that were differently expressed in the IF of leiomyomas compared with that of the normal myometrium were identified (13). The present study continued to investigate different abundant proteins likely to be associated with tumor development in the IF.

The adoption of 18-cm pH 3-10 NL strips with polyacrylamide gels of 19x20 $\mathrm{cm}$ in size instead of 7-cm pH 3-10 strips with polyacrylamide gels of $8 \times 8 \mathrm{~cm}$ in size markedly increased the spot number. This allowed the identification of 22 proteins that had not been previously identified in the leiomyoma IF, while PGAM1 had never previously been associated 
with leiomyoma. IPA analysis predicted that 17 out of the 25 proteins are associated with cellular migration and proliferation. In addition, the abundance of the proteins identified in our previous study (DES, TAGLN, SERPINA1, LMNA, ACTN1, PRDX2 and HSPA1A) is in line with the results of the present study on the leiomyoma IF. Furthermore, the levels of CRABP2, CALM1, LDHB, SERPINA1 and DES were confirmed to be upregulated, while those of TAGLN, LMNA and ACTN1 were downregulated, both in the proteome $(12,13)$ and in the IF. Seven of these proteins (LUM, PRDX1, CRABP2, CALR, ANXA2, ALDOA and PGAM1) were validated by western blotting, thus confirming the 2-DE data.

An inadequate vascularization during tumor growth results in microenvironmental stress, which induces the activation of a range of stress response pathways, including the unfolding protein response (UPR) (18). UPR activation is required for tumor cell growth under hypoxic conditions (18). CALR is a protein involved in the UPR pathway. It participates in several biological processes, including cell adhesion, gene expression, RNA stability and regulation of $\mathrm{Ca}^{2+}$ homeostasis $(19,20)$. Cell stress increases the concentration of nitric oxide, inducing the release of CALR from cells in the extracellular space (21). This may be associated with alterations in the viscosity and elasticity of the ECM.

The presence of adenylyl cyclase-associated protein 1 (CAP1) in the extracellular environment was documented in the urine of patients with systemic autoimmune diseases, and was identified as an efficient substrate of gelatinase B/matrix metallopeptidase (MMP)-9 (22). Degradation of CAP1 by MMP-9 is important for tumor proliferation (22), as it may accelerate ECM accumulation, thereby promoting tumor growth.

Inflammatory and pro-inflammatory factors serve a key role in cancer development with the involvement of the transcription factor nuclear factor $(\mathrm{NF})-\kappa \mathrm{B}$, which also has a crucial role in cell growth, tumorigenesis and apoptosis (23). Several pro-inflammatory factors, including NF- $\kappa$ B, interleukin-1 $\beta$, tumor necrosis factor- $\alpha$ and cyclooxygenase-2, are involved in the cellular proliferation and growth of leiomyomas (23). PRDX1 and PRDX2 are released by cells in the presence of pro-inflammatory factors. Their release stimulates the production of inflammatory cytokines, thus increasing the proliferative effect, which serves a role in matrix production $(24,25)$.

CRABP2 is a key protein for the binding and transport of retinoic acid (26). Increasing levels of retinoic acid induce the overexpression of MMP-11, which degrades insulin-like growth factor-binding proteins, and may result in cell proliferation, decreased apoptosis and acceleration of ECM accumulation, thereby promoting the growth of uterine leiomyomas (27).

The present study identified two glycolytic enzymes (ALDOA and PGAM1) with different abundance in the leiomyoma IF. This further confirms that glycolytic enzymes can be secreted by cells, attest metabolic dysregulation in leiomyomas (12) and perform non-enzymatic activities such as structural or regulatory functions at their extracellular localization (28).

ANXA2 is a $\mathrm{Ca}^{2+}$-dependent phospholipid binding protein able to bind plasminogen and mediate the localized generation of plasmin (29). The present study has shown that the IF of leiomyomas is characterized by a lower abundance compared with the myometrium, of ALDOA and ANXA2, which could be associated with a decreased proteolytic degradation of the ECM in leiomyomas.

HSPA1A is a member of the Hsp70 family (30). In cooperation with other chaperones, HSPA1A mediates the folding of newly translated polypeptides in the cytosol, as well as within organelles (31). Once secreted, HSPA1A participates in paracrine or autocrine interactions with adjacent cell surfaces, controlling the expression of several genes associated with apoptosis and cell migration $(32,33)$.

It is well known that leiomyomas are characterized by an abnormal ECM production (6). Proteoglycan components of the ECM such as LUM bind to integrin, activating the integrin signaling pathway, and leading to cytoskeletal rearrangement and cell motility (34). Physiologically, LUM is involved in several biological processes, including the glycosaminoglycan metabolic process, angiogenesis, cell proliferation, migration, adhesion and ECM organization (35). Leiomyoma growth may be closely associated with secreted LUM, which is in turn associated with cell motility and growth.

IPA revealed remarkable data on the interaction among proteins. Several proteins identified in the present study (including PRDX2, ANXA1, ANXA2, HSPA1A, filamin A $\alpha$, CALR, calmodulin 1 and talin 1) interact substantially with extracellular signal-regulated kinase-1/2, platelet-derived growth factor-BB and calpain, which serve a key role in cell growth, adhesion, survival and differentiation $(36,37)$.

In conclusion, the comparative proteomic approach conducted in the present study identified a number of proteins associated with cellular migration and proliferation with different abundance in the IF. Secreted proteins may be involved in mechanisms associated with leiomyoma development. Further studies will be required to investigate the role of these proteins in the leiomyoma IF and their association with tumor development, in order to identify novel therapeutic targets and develop new pharmacological approaches for the treatment of leiomyoma.

\section{Acknowledgements}

The authors acknowledge the help of Dr Emmanouil Athanasakis (Medical Genetics Department, Institute for Maternal and Child Health-IRCCS 'Burlo Garofolo', Trieste, Italy) for data acquisition. The present study was internally funded by the Institute for Maternal and Child Health-IRCCS 'Burlo Garofolo' of Trieste, Italy (grant no. RC 19/08).

\section{References}

1. Hashimoto K, Azuma C, Kamiura S, Kimura T, Nobunaga T, Kanai T, Sawada M, Noguchi S and Saji F: Clonal determination of uterine leiomyomas by analyzing differential inactivation of the X-chromosome-linked phosphoglycerokinase gene. Gynecol Obstet Invest 40: 204-208. 1995.

2. Haney AF: Clinical decision making regarding leiomyomata: What we need in the next millennium. Environ Health Perspect 108 (Suppl 5): S835-S839, 2000.

3. Borahay MA, Al-Hendy A, Kilic GS and Boehning D: Signaling pathways in leiomyoma: Understanding pathobiology and implications for therapy. Mol Med 21: 242-256. 2015.

4. Catherino WH and Malik M: Uterine leiomyomas express a molecular pattern that lowers retinoic acid exposure. Fertil Steril 87: 1388-1398, 2007. 
5. Arslan AA, Gold LI, Mittal K, Suen TC, BelitskayaLevy I, Tang MS and Toniolo P: Gene expression studies provide clues to the pathogenesis of uterine leiomyoma: New evidence and a systematic review. Hum Reprod 20: 852-863, 2005.

6. Malik M and Catherino WH: Novel method to characterize primary cultures of leiomyoma and myometrium with the use of confirmatory biomarker gene arrays. Fertil Steril 87: 1166-1172, 2007.

7. Gromov P, Gromova I, Olsen CJ, Timmermans-Wielenga V, Talman ML, Serizawa RR and Moreira JM: Tumor interstitial fluid-a treasure trove of cancer biomarkers. Biochim Biophys Acta 1834: 2259-2270, 2103.

8. Spano D and Zollo M: Tumor microenvironment: A main actor in the metastasis process. Clin Exp Metastasis 29: 381-395, 2012

9. Norian JM, Owen CM, Taboas J, Korecki C, Tuan R, Malik M, Catherino WH and Segars JH: Characterization of tissue biomechanics and mechanical signaling in uterine leiomyoma. Matrix Biol 31: 57-65, 2012

10. Lv J, Zhu X, Dong K, Lin Y, Hu Y and Zhu C: Reduced expression of 14-3-3 gamma in uterine leiomyoma as identified by proteomics. Fertil Steril 90: 1892-1898, 2008.

11. Lemeer S, Gholami AM, Wu Z and Kuster B: Quantitative proteome profiling of human myoma and myometrium tissue reveals kinase expression signatures with potential for therapeutic intervention. Proteomics 15: 356-364, 2015.

12. Ura B, Scrimin F, Arrigoni G, Franchin C, Monasta L and Ricci G: A proteomic approach for the identification of up-regulated proteins involved in the metabolic process of the leiomyoma. Int J Mol Sci 17: 540, 2016.

13. Ura B, Scrimin F, Arrigoni G, Athanasakis E, Aloisio M, Monasta L and Ricci G: Abnormal expression of leiomyoma cytoskeletal proteins involved in cell migration. Oncol Rep 35: 3094-3100, 2016

14. Ura B, Scrimin F, Zanconati F, Arrigoni G, Monasta L, Romano A Banco R, Zweyer M, Milani D and Ricci G: Two-dimensional gel electrophoresis analysis of the leiomyoma interstitial fluid reveals altered protein expression with a possible involvement in pathogenesis. Oncol Rep 33: 2219-2226, 2015.

15. Gromov P, Gromova I, Bunkenborg J, Cabezon T, Moreira JM, Timmermans-Wielenga V, Roepstorff P, Rank F and Celis JE: Up-regulated proteins in the fluid bathing the tumour cell microenvironment as potential serological markers for early detection of cancer of the breast. Mol Oncol 4: 65-89, 2010.

16. Mischiati C, Ura B, Roncoroni L, Elli L, Cervellati C, Squerzanti M, Conte D, Doneda L, Polverino de Laureto P, de Franceschi G, et al: Changes in protein expression in two cholangiocarcinoma cell lines undergoing formation of multicellular tumor spheroids in vitro. PLoS One 10: e0118906, 2015.

17. Li S and McLachlan JA: Estrogen-associated genes in uterine leiomyoma. Ann N Y Acad Sci 948: 112-120, 2001.

18. Desiniotis A and Kyprianou N: Significance of talin in cancer progression and metastasis. Int Rev Cell Mol Biol 289: 117-147, 2011.

19. Vandewynckel YP, Laukens D, Geerts A, Bogaerts E, Paridaens A, Verhelst X, Janssens S, Heindryckx $F$ and Van Vlierberghe $\mathrm{H}$ : The paradox of the unfolded protein response in cancer. Anticancer Res 33: 4683-4694, 2013.

20. Totary-Jain H, Naveh-Many T, Riahi Y, Kaiser N, Eckel J and Sasson S: Calreticulin destabilizes glucose transporter-1 mRNA in vascular endothelial and smooth muscle cells under high-glucose conditions. Circ Res 97: 1001-1008, 2005.

21. Tarr JM, Young PJ, Morse R, Shaw DJ, Haigh R, Petrov PG, Johnson SJ, Winyard PG and Eggleton P: A mechanism of release of calreticulin from cells during apoptosis. J Mol Biol 401: 799-812, 2010
22. Cauwe B, Martens E, Van den Steen PE, Proost P, Van Aelst I, Blockmans D and Opdenakker G: Adenylyl cyclase-associated protein-1/CAP1 as a biological target substrate of gelatinase B/MMP-9. Exp Cell Res 314: 2739-2749, 2008.

23. Plewka A, Madej P, Plewka D, Kowalczyk A, Miskiewicz A, Wittek P, Leks T and Bilski R: Immunohistochemical localization of selected pro-inflammatory factors in uterine myomas and myometrium in women of various ages. Folia Histochem Cytobiol 51: 73-83, 2013.

24. Mullen L, Hanschmann EM, Lillig CH, Herzenberg LA and Ghezzi P: Cysteine oxidation targets peroxiredoxins 1 and 2 for exosomal release through a novel mechanism of redox-dependent secretion. Mol Med 13: 98-108, 2015

25. Kubota D, Mukaihara K, Yoshida A, Tsuda H, Kawai A and Kondo T: Proteomics study of open biopsy samples identifies peroxiredoxin 2 as a predictive biomarker of response to induction chemotherapy in osteosarcoma. J Proteomics 91: 393-404, 2013.

26. Aström A, Pettersson U and Voorhees JJ: Structure of the human cellular retinoic acid-binding protein II gene. Early transcriptional regulation by retinoic acid. J Biol Chem 267: 25251-25255, 1992

27. Zaitseva M, Vollenhoven BJ and Rogers PA: Retinoic acid pathway genes show significantly altered expression in uterine fibroids when compared with normal myometrium. Mol Hum Reprod 13: 577-585, 2007.

28. Capello M, Ferri-Borgogno S, Cappello P and Novelli F: $\alpha$-Enolase: A promising therapeutic and diagnostic tumor target. FEBS J 278: 1064-1074, 2011.

29. Valapala M, Maji S, Borejdo J and Vishwanatha JK: Cell surface translocation of annexin A2 facilitates glutamate-induced extracellular proteolysis. J Biol Chem 289: 15915-15926, 2014.

30. Hageman J, Vos MJ, van Waarde MA and Kampinga HH: Comparison of intra-organellar chaperone capacity for dealing with stress-induced protein unfolding. J Biol Chem 282: 34334-34345, 2007.

31. Chen Z, Barbi J, Bu S, Yang HY, Li Z, Gao Y, Jinasena D, Fu J, Lin F, Chen C, et al: The ubiquitin ligase Stub1 negatively modulates regulatory $\mathrm{T}$ cell suppressive activity by promoting degradation of the transcription factor Foxp3. Immunity 39: 272-285, 2013.

32. Mambula SS, Stevenson MA, Ogawa K and Calderwood SK: Mechanisms for Hsp70 secretion: Crossing membranes without a leader. Methods 43: 168-175, 2007.

33. Daniels GA, Sanchez-Perez L, Diaz RM, Kottke T, Thompson J, Lai M, Gough M, Karim M, Bushell A and Chong H: A simple method to cure established tumors by inflammatory killing of normal cells. Nat Biotechnol 22: 1125-1132, 2004.

34. San Martin S, Soto-Suazo M, De Oliveira SF, Aplin JD, Abrahamsohn P and Zorn TM: Small leucine-rich proteoglycans (SLRPs) in uterine tissues during pregnancy in mice. Reproduction 125: 585-595, 2003.

35. Nikitovic D, Papoutsidakis A,Karamanos NK and Tzanakakis GN: Lumican affects tumor cell functions, tumor-ECM interactions, angiogenesis and inflammatory response. Matrix Biol 35: 206-214, 2014.

36. Lorenz K, Schmitt JP, Schmitteckert EM and Lohse MJ: A new type of ERK1/2 autophosphorylation causes cardiac hypertrophy. Nat Med 15: 75-83, 2009.

37. Richard I, Broux O, Allamand V, Fougerousse F, Chiannilkulchai N, Bourg N, Brenguier L, Devaud C, Pasturaud P, Roudaut C, et al: Mutations in the proteolytic enzyme calpain 3 cause limb-girdle muscular dystrophy type 2A. Cell 81: 27-40, 1995. 